\title{
CÂNCER DE COLO UTERINO: CONHECIMENTO E COMPORTAMENTO DE MULHERES PARA PREVENÇÃO
}

\author{
Cervical cancer: knowledge and behavior of women for \\ prevention
}

Cáncer de cuello uterino: conocimiento y conducta de mujeres
hacia la prevención

Artigo Original

\section{RESUMO}

Objetivo: Analisar o conhecimento das mulheres em relação à prevenção do câncer de colo de útero e os fatores dificultadores acerca da realização da prática do exame preventivo. Métodos: Trata-se de um estudo do tipo observacional, de corte transversal e descritivo, com 110 mulheres entre 25 e 64 anos, atendidas em uma unidade de saúde, entre os meses de janeiro e março de 2014. Coletaram-se dados sociodemográficos e econômicos, aspectos ginecológicos e comportamento sobre o exame. Analisaram-se os dados através da estatística descritiva, apresentando valores absolutos e relativos. Resultados: Dados referentes ao significado do câncer uterino mostraram que 65 (59,1\%) desconheciam seu significado, 69 (62,7\%) sabiam como preveni-lo, 104 (94,5\%) já realizaram o Papanicolau, 59 (53,6\%) realizaram o Papanicolau há 1 ano, $62(56,4 \%)$ realizam o exame preventivo anualmente e $88(80 \%)$ sabiam a importância dessa realização. Quanto aos fatores encontrados referentes à dificuldade na realização do exame preventivo, 49 (44,5\%) relataram ser a vergonha o fator mais impactante. Conclusão: Apesar de a maioria das mulheres realizar o exame periodicamente, muitas desconhecem a sua verdadeira finalidade, sentindo-se envergonhadas e constrangidas durante a realização do exame.

Descritores: Enfermagem; Saúde da Mulher; Neoplasia do Colo; Papanicolaou.

\section{ABSTRACT}

Objective: To analyze the knowledge of women regarding prevention of cervical cancer and the factors hindering the performance of screening tests. Methods: This is a descriptive observational cross-sectional study conducted with 110 women aged 25-64 years treated at a health center between January and March 2014. We collected sociodemographic and economic data and information on gynecological aspects and behavior regarding the exam. Data were analyzed using descriptive statistics with absolute and relative values. Results: Data relating to the meaning of cervical cancer showed that 65 (59.1\%) women were unaware of its meaning, 69 (62.7\%) knew how to prevent it, 104 (94.5\%) had already done Pap-smear test, 59 (53.6\%) had done the Pap-smear test in the past year, 62 (56.4\%) do the screening test annually, 88 (80\%) knew the importance of the screening test. Regarding the factors related to the difficulty in performing the screening test, most women 49 (44.5\%) reported the shame as the most impactful factor. Conclusion: Although most women do the test regularly, many are unaware of its real purpose and feel ashamed and embarrassed during the test.

Descriptors: Nursing; Women's Health; Colonic Neoplasms; Papanicolau Test.

1) Universidade Federal de Alagoas - UFAL - Maceió (AL) - Brasil 


\section{RESUMEN}

Objetivo: Analizar el conocimiento de las mujeres sobre la prevención del cáncer de cuello uterino y los factores que dificultan la realización de la práctica del examen preventivo. Métodos: Se trata de un estudio del tipo observacional, de corte transversal y descriptivo con 110 mujeres entre 25 y 64 años asistidas en una unidad de salud entre los meses de enero y marzo de 2014. Se recogieron los datos sociodemográficos y económicos, los aspectos ginecológicos y de la conducta del examen. Los datos fueron analizados a través de la estadística descriptiva con valores absolutos y relativos. Resultados: Los datos referentes al significado del cáncer de cuello uterino mostraron que 65 (59,1\%) mujeres desconocían su significado, 69 (62,7\%) sabian cómo prevenirlo, 104 (94,5\%) ya habian realizado el Papanicolaou, 59 (53,6\%) realizaron el Papanicolaou en el último año, 62 (56,4\%) realizan el examen preventivo cada año y 88 (80\%) sabian de la importancia de su realización. Sobre los factores relativos a la dificultad de realización del examen preventivo, 49 (44,5\%) relataron la vergüenza como el factor más importante. Conclusión: Aunque la mayoría de las mujeres realizan el examen con periodicidad muchas desconocen su verdadera finalidad, sintiéndose avergonzadas y constreñidas durante su realización.

Descriptores: Enfermeria; Salud de la Mujer; Neoplasia del Colon; Prueba de Papanicolaou.

\section{INTRODUÇÃO}

O câncer de colo uterino (CCU) é tido como afecção progressiva e caracterizado por alterações intraepiteliais cervicais que podem se desenvolver para um estágio invasivo ao longo de uma a duas décadas. Possuindo etapas bem definidas e de lenta evolução, o câncer de colo de útero pode ser interrompido a partir de um diagnóstico precoce e tratamento oportuno a custos reduzidos ${ }^{(1)}$.

Mesmo assim os índices são alarmantes, pois aproximadamente 530 mil mulheres irão desenvolver o câncer de colo do útero por ano no mundo, sendo o terceiro tipo de câncer mais comum entre o sexo feminino, responsável pelo óbito de 275 mil mulheres por ano. O principal fator de risco para o desenvolvimento de lesões intraepiteliais de alto grau e do câncer do colo do útero é a infecção pelo papilomavírus humano (HPV). Existem hoje 13 tipos de HPV reconhecidos como oncogênicos pela Agência Internacional para Pesquisa sobre o Câncer (IARC). Desses, os mais comuns são o HPV16 e o HPV18 $8^{(2,3)}$.

Apesar de ser considerada uma condição necessária, a infecção pelo HPV não representa uma causa suficiente para o surgimento dessa neoplasia. Além de aspectos relacionados à própria infecção pelo HPV (tipo e carga viral, infecção única ou múltipla), outros fatores ligados à imunidade, à genética e ao comportamento sexual parecem influenciar os mecanismos ainda incertos, determinando a regressão ou a persistência da infecção, e também a progressão para lesões precursoras ou câncer ${ }^{(4)}$.

A idade também interfere nesse processo. A maioria das infecções por HPV em mulheres com menos de 30 anos regride espontaneamente, ao passo que acima dessa idade a persistência é mais frequente. O tabagismo aumenta o risco para o desenvolvimento do câncer do colo do útero, proporcionalmente ao número de cigarros fumados por dia e ao início em idade precoce ${ }^{(4)}$.

O câncer de colo do útero é uma doença de crescimento lento e silencioso. Existe uma fase pré-clínica, sem sintomas, com transformações intraepiteliais progressivas importantes, em que a detecção de possíveis lesões precursoras acontece por meio da realização periódica do exame preventivo do colo do útero. Essa patologia vai progredindo por anos, antes de atingir o estágio invasor da doença, quando a cura se torna mais difícil.

Nessa fase, os principais sintomas são sangramento vaginal, corrimento e dor. O rastreamento do câncer do colo do útero representa um processo complexo em múltiplas etapas a compor: aplicação do exame de rastreamento, identificação dos casos positivos (suspeitos de lesões precursoras ou câncer), confirmação diagnóstica e tratamento ${ }^{(3,5)}$.

No Brasil, a principal estratégia utilizada para a detecção precoce/rastreamento do câncer de colo do útero é a realização da coleta de material para exames citopatológicos cervicovaginal e microflora, conhecido popularmente como exame preventivo do colo do útero, exame de Papanicolaou, citologia oncótica e Paptest. A periodicidade de realização do exame preventivo, estabelecida pelo Ministério da saúde do Brasil em 1988, permanece atual e está de acordo com as recomendações dos principais programas internacionais. O exame citopatológico deve ser realizado em mulheres de 25 a 60 anos de idade, uma vez por ano e, após dois exames consecutivos negativos, a cada três anos ${ }^{(4,5)}$.

As práticas de Prevenção do Câncer do Colo do Útero (PCCU), ainda hoje, representam um importante desafio de saúde pública. As razões para isso devem-se aos fatores culturais, sociais, econômicos e comportamentais, bem como à própria organização dos serviços públicos de saúde.

Além desses fatores, a demora das mulheres em realizar o exame ou a ausência deste no serviço pode estar associada ao modo como a usuária percebe o exame preventivo. A percepção das mulheres é influenciada pelos valores, pela cultura, pela raça, pelas experiências vividas, crenças, expectativas de vida e ideias pré-concebidas construídas ao longo da vida ${ }^{(6)}$. 
Vencer as barreiras para uma melhor adesão da mulher ao exame preventivo significa dar atenção aos relatos e às experiências de quem a ele se submete. Portanto, o presente estudo se justifica pelo fato de que a cada ano mais mulheres adoecem por falta de conhecimento sobre como prevenir o câncer de colo do útero, além do fato de o profissional de saúde não estar preparado para lidar com situações que colocam a mulher em constrangimento.

Para mudar essa realidade é preciso que, através das informações apresentadas, os profissionais de saúde, dentre eles o enfermeiro, consigam planejar e orientar os serviços de prevenção com vistas à promoção da saúde. Esse é o primeiro caminho para a diminuição da incidência desse tipo de câncer( ${ }^{(6)}$.

Diante do exposto, o estudo teve como objetivo analisar o conhecimento das mulheres em relação à prevenção do câncer de colo de útero e os fatores dificultadores acerca da realização da prática do exame preventivo.

\section{MÉTODOS}

Trata-se de um estudo observacional, de corte transversal e descritivo, desenvolvido na Unidade de Saúde Dr. Hamilton Falcão, no Bairro Benedito Bentes, do município de Maceió-AL. A amostra foi constituída por 110 mulheres na faixa etária de 25 a 64 anos que compareceram à Unidade de Saúde Dr. Hamilton Falcão para a realização do exame preventivo do câncer de colo uterino.

O cálculo amostral foi baseado na quantidade de mulheres atendidas na referida unidade de saúde e na prevalência da doença $(56 \%)^{(7)}$, com precisão absoluta de $10 \%$ e nível de significância de $5 \%$. Realizou-se a seleção das mulheres de forma consecutiva, baseando-se nos seguintes critérios de inclusão: mulher na faixa etária de 25 a 64 anos que realizaram o exame citopatológico e se dispuserem a colaborar voluntariamente com este estudo.

Fez-se a coleta dos dados nos meses de janeiro a março de 2014, por meio de um roteiro pré-elaborado contendo dados sociodemográficos e econômicos (informações sobre idade, estado civil, escolaridade e profissão), dados ginecológicos (realização do exame, sexarca e gestações anteriores) e dados referentes ao conhecimento e comportamento das mulheres sobre a prevenção e o significado do câncer de colo uterino. Realizou-se análise descritiva e os resultados foram apresentados em números absolutos e frequência simples.

O estudo foi aprovado pelo Comitê de Ética em Pesquisa da Universidade Federal de Alagoas (parecer $n^{\circ}$. 22885113.6.0000.5013) e seguiu a Resolução 466/12 do Conselho Nacional de Saúde. As participantes assinaram um termo de consentimento livre e esclarecido concordando com sua participação voluntária.

\section{RESULTADOS}

Os dados sociodemográficos e econômicos descrevem uma amostra em que $35(31,8 \%)$ das mulheres tinham entre 25 e 34 anos, 52 (47,3\%) eram casadas, 77 (70\%) não

Tabela I - Distribuição dos dados sociodemográficos e econômicos das mulheres atendidas em uma unidade básica. Maceió-AL, 2014.

\begin{tabular}{|c|c|c|}
\hline Variáveis & $\mathbf{n}$ & $\%$ \\
\hline \multicolumn{3}{|l|}{ Idade } \\
\hline $25-34$ anos & 35 & 31,8 \\
\hline $35-44$ anos & 32 & 29,1 \\
\hline $45-55$ anos & 30 & 27,3 \\
\hline+55 anos & 13 & 11,8 \\
\hline \multicolumn{3}{|l|}{ Estado Civil } \\
\hline Casada & 52 & 47,3 \\
\hline União estável & 30 & 27,3 \\
\hline Divorciada & 9 & 8,2 \\
\hline Solteira & 9 & 8,2 \\
\hline Viúva & 10 & 9,0 \\
\hline \multicolumn{3}{|l|}{ Escolaridade } \\
\hline Analfabeta & 13 & 11,8 \\
\hline Ensino fundamental incompleto & 47 & 42,7 \\
\hline Ensino fundamental completo & 8 & 7,3 \\
\hline Ensino médio incompleto & 9 & 8,2 \\
\hline Ensino médio completo & 29 & 26,4 \\
\hline Ensino superior & 4 & 3,6 \\
\hline \multicolumn{3}{|l|}{ Profissão } \\
\hline Dona de casa & 72 & 65,5 \\
\hline Garçonete & 1 & 0,9 \\
\hline Técnica de nutrição & 1 & 0,9 \\
\hline Servidora pública & 3 & 2,7 \\
\hline Cuidadora & 3 & 2,7 \\
\hline Auxiliar de educação & 1 & 0,9 \\
\hline Empregada doméstica & 17 & 15,5 \\
\hline Cabeleireira & 2 & 1,8 \\
\hline Técnica de enfermagem & 2 & 1,8 \\
\hline Comerciante & 4 & 3,6 \\
\hline Frentista & 1 & 0,9 \\
\hline Operadora de caixa & 1 & 0,9 \\
\hline Auxiliar de auditoria & 1 & 0,9 \\
\hline Ajudante de pedreiro & 1 & 0,9 \\
\hline \multicolumn{3}{|l|}{ Renda Familiar } \\
\hline$<1$ salário mínimo & 14 & 12,7 \\
\hline 1 a 2 salários mínimos & 81 & 73,7 \\
\hline$>2$ salários mínimos & 15 & 13,6 \\
\hline
\end{tabular}


atingiram o ensino médio, $72(65,5 \%)$ eram donas de casa e $81(73,6 \%)$ tinham renda familiar de até 2 salários mínimos (Tabela I).

Com relação aos fatores de risco para o câncer de colo uterino, identificou-se que $73(66,4 \%)$ das entrevistadas tiveram entre uma e três gestações, 96 (87,3\%) iniciaram atividades sexuais com idade entre 12 e 20 anos, e 67 $(60,9 \%)$ das mulheres entrevistadas tiveram de dois a sete parceiros sexuais. Entre as entrevistadas, as doenças sexualmente transmissíveis (DST) foram relatadas por 29 (26,4\%) das mulheres, 57 (51,7\%) disseram ser DST do tipo corrimento, e apenas 41 (37,9\%) citaram o HPV (Tabela II).

Tabela II - Distribuição das entrevistadas em relação aos fatores de risco para o câncer de colo uterino. Maceió-AL, 2014.

\begin{tabular}{|c|c|c|}
\hline Variáveis & $\mathbf{n}$ & $\%$ \\
\hline \multicolumn{3}{|c|}{ Gestações (número) } \\
\hline Nenhuma & 09 & 8,2 \\
\hline 1 a 3 & 73 & 66,4 \\
\hline 4 a 7 & 19 & 17,2 \\
\hline Mais de 8 & 09 & 8,2 \\
\hline \multicolumn{3}{|c|}{ Parceiros sexuais (número) } \\
\hline 1 a 3 & 67 & 60,9 \\
\hline 4 a 7 & 24 & 21,8 \\
\hline 7 a 10 & 04 & 3,6 \\
\hline 10 a 20 & 02 & 1,8 \\
\hline 21 a 30 & 10 & 9,1 \\
\hline$>30$ & 03 & 2,7 \\
\hline \multicolumn{3}{|l|}{ Sexarca } \\
\hline$<12$ anos & 01 & \\
\hline 12 a 16 anos & 01 & 0,9 \\
\hline 17 a 20 anos & $\begin{array}{l}44 \\
52\end{array}$ & $\begin{array}{l}40,0 \\
47,3\end{array}$ \\
\hline$>21$ anos & $\begin{array}{l}52 \\
13\end{array}$ & \\
\hline \multicolumn{3}{|c|}{ Portadoras de DST } \\
\hline Sim & 29 & 26,4 \\
\hline Não & 81 & 73,6 \\
\hline \multicolumn{3}{|l|}{ Tipos de DST } \\
\hline Úlceras & 11 & 10,3 \\
\hline Corrimentos & 57 & 51,8 \\
\hline HPV & 41 & 37,3 \\
\hline
\end{tabular}

DST: Doenças sexualmente transmissíveis; HPV: Papilomavírus Humano.
Quando questionadas se sabiam o que significa o câncer do colo do útero, $65(59,1 \%)$ responderam não. Entre aquelas que referiram saber o significa o câncer de colo uterino, 28 $(44,4 \%)$ associaram essa neoplasia a ferida, lesão, tumor ou deformação no útero como significado para doença. Das mulheres entrevistadas, $68(62,7 \%)$ relataram saber como prevenir o câncer de colo uterino, entre as quais $36(52,9 \%)$ citaram o preservativo como principal meio de prevenção, seguido do exame preventivo de Papanicolau, com 28 $(43,5 \%)$ (Tabela III).

Quanto ao exame preventivo de câncer de colo uterino, $104(94,5 \%)$ já tinham realizado o exame pelo menos uma vez; destas, $59(56,73 \%)$ realizaram o último exame há um

Tabela III - Distribuição das entrevistadas segundo o conhecimento sobre o câncer de colo uterino. Maceió-AL, 2014.

\begin{tabular}{lcc}
\hline Variáveis & n & \% \\
\hline Conhecimento & & \\
$\quad$ Sim & 45 & 40,9 \\
$\quad$ Não & 65 & 59,1 \\
$\quad$ Significado & & \\
$\quad$ Ferida/lesão/deformação & 28 & 44,4 \\
$\quad$ Doença/inflamação/doença venérea & 17 & 55,6 \\
Prevenção & & \\
$\quad$ Sim & 68 & 61,8 \\
$\quad$ Não & 42 & 38,2 \\
Métodos de Prevenção & 03 & 3,6 \\
$\quad$ Outros & 28 & 43,5 \\
$\quad$ Exame Papanicolau & 36 & 52,9 \\
$\quad$ Preservativos &
\end{tabular}

Tabela IV - Dificuldades encontradas para realização do exame preventivo de colo uterino. Maceió-AL, 2014.

\begin{tabular}{lcc}
\hline Dificuldades & n & $\mathbf{\%}$ \\
\hline Nenhuma & 41 & 37,3 \\
Vergonha & 49 & 44,5 \\
Medo & 4 & 3,6 \\
Dificuldade de acesso & 8 & 7,3 \\
Descaso & 6 & 5,4 \\
Falta de tempo & 2 & 1,9 \\
\hline
\end{tabular}


ano. A importância de realizar o exame de Papanicolau foi reconhecida por $88(80 \%)$ das participantes. Com relação às dificuldades para realizar do exame, o maior sentimento percebido foi a vergonha durante o procedimento, relatada por $49(44,5 \%)$ mulheres (Tabela IV).

\section{DISCUSSÃO}

A maioria das mulheres que participou deste estudo encontra-se em uma faixa etária compreendida entre $25 \mathrm{e}$ 44 anos. Para o Instituto Nacional de Câncer José Alencar da Silva (INCA) ${ }^{(7)}$, a incidência do câncer de colo de útero aumenta nas mulheres entre 30 e 39 anos de idade, e atinge seu pico na quinta ou sexta década de vida. Antes dos 25 anos, prevalecem as infecções por HPV e as lesões de baixo grau, que regredirão espontaneamente na maioria dos casos e, portanto, podem ser apenas acompanhadas conforme recomendações clínicas.

Após os 65 anos, por outro lado, com os exames preventivos regulares e normais, o risco de desenvolvimento do câncer cervical é reduzido. Porém, esse quadro vem se modificando aos poucos e o aparecimento de lesões precursoras está ocorrendo cada vez mais precocemente, devido à iniciação antecipada das atividades sexuais associada aos demais fatores de risco, tais como multiparidade, tabagismo, vários parceiros e infecções sexualmente transmissíveis ${ }^{(8)}$.

Houve um expressivo número de mulheres casadas ou em união estável na população em estudo. Tais tipos de união conjugal podem conduzir as esposas a uma maior exposição, principalmente a doenças infecciosas do trato genital transmitidas por relações sexuais, devido à confiança na fidelidade de seus parceiros, que as levam a não utilizarem nenhum método de prevenção( ${ }^{(8)}$.

O câncer do colo do útero é mais frequente em mulheres de classes sociais mais baixas e com menor nível de escolaridade, pois o acesso reduzido à informação sobre cuidados de saúde pode estar relacionado ao baixo nível de escolaridade, fato que pode refletir diretamente na demanda dos exames preventivos, na melhor compreensão das informações sobre as doenças e na necessidade de atitudes favoráveis à detecção precoce das neoplasias. As mulheres com baixo grau de instrução têm maior risco de desenvolver câncer do colo uterino, com uma relação indireta entre o grau de instrução e o risco do diagnóstico avançado desse tumor ${ }^{(9)}$.

Em consonância com esse fato, um estudo detectou que mulheres com baixa renda são mais vulneráveis a infecções sexualmente transmissíveis (IST's), pois apresentam baixo poder de argumentação e negociação com o parceiro. Por outro lado, aquelas que apresentam maior escolaridade são provavelmente mais conscientes de seus direitos e têm maior poder de praticar sexo seguro ${ }^{(10)}$.
Além da escolaridade, a ocupação é também determinante para o câncer de colo uterino, pois mulheres que trabalham fora de casa apresentam proporções mais elevadas de atitudes adequadas em relação ao exame Papanicolau, bem como maior acesso à informação nos contatos com outras trabalhadoras. Isso pode estimular práticas preventivas de saúde. Em contrapartida, mulheres que trabalham exclusivamente em casa têm menos autonomia em tomar decisões relativas à saúde ${ }^{(8,11)}$.

A ocorrência de partos antes dos 20 anos em mulheres com DNA do HPV é um fator de risco consistente para o desenvolvimento da doença. Esse risco dobra nas mulheres com quatro filhos, quando comparado com aquelas de um ou nenhum filho. Mulheres com sete ou mais gestações apresentam um risco 3,9 vezes maior se comparadas com mulheres nulíparas ${ }^{(12)}$. A explicação possível seria o trauma à cérvice durante o parto, ou o aumento da susceptibilidade à infecção resultante da imunossupressão, influências hormonais e deficiências dietéticas, embora ainda não seja suficientemente comprovada ${ }^{(12)}$.

A precocidade da coitarca (10-14 anos) e da gravidez (até 19 anos) são fatores de risco para o $\mathrm{CCU}$, pois na adolescência a metaplasia se intensifica e o coito aumenta a probabilidade de transformação atípica, potencializando as chances de alteração celular, favorecendo a infecção pelo HPV e outros microrganismos. Soma-se a isso a maior exposição aos seguintes fatores de risco: multiplicidade de parceiros, parceiros de risco, exposição às ISTs e ação do líquido seminal. Assim, aumenta-se o risco de adquirir uma lesão precursora que poderá se desenvolver para um câncer de colo uterino ${ }^{(8,10)}$.

Vários autores apontaram aumento na incidência de lesões cervicais por HPV em mulheres cujo número de parceiros sexuais foi maior que dois ${ }^{(8,12,13)}$, com a incidência das lesões precursoras diretamente relacionada ao número de parceiros sexuais masculinos, tendo aquelas com mais de dois parceiros um risco aumentado em 5 vezes. Sugere-se o homem como reservatório de DNA do HPV, armazenandoos no órgão reprodutor e disseminando-os ${ }^{(12)}$.

Outro fator de risco destacado neste estudo é a presença de ISTs no grupo de voluntárias. Mulheres com ISTs apresentam cinco vezes mais alterações celulares do colo do útero. Isso ocorre devido à possibilidade de a maioria dessas alterações provocar inflamação e/ou ulceração genital, facilitando a disseminação de outras DST, incluindo o HPV ${ }^{(10)}$.

O nível de conhecimento sobre a doença foi considerado baixo entre as mulheres no geral, pois a procura pelos serviços ocorre em virtude da presença de alguma queixa, e não pela prevenção. Além disso, pouco se sabe sobre os sintomas e sinais do câncer de colo uterino, sendo as informações provenientes da população. 
A falta de conhecimento traz como consequência a baixa conscientização sobre o significado, a importância do exame de Papanicolau e o restrito acesso à assistência de saúde. Esse fato, em parte, é responsável pelo número expressivo de mulheres que nunca realizaram o exame e acabam descobrindo a doença já em estágio avançado ${ }^{(11,13)}$. O câncer do colo uterino pode ser curado quando descoberto precocemente, entretanto, ainda há mulheres desenvolvendo esse tipo de câncer e morrendo no Brasil pelo fato de desconhecerem a finalidade do exame ${ }^{(13,14)}$.

Mesmo com a implantação de programas pelo Ministério da Saúde e a ampla divulgação das informações a respeito do exame preventivo na rede básica de saúde, as mulheres têm pouca clareza ou nenhum conhecimento do significado da prevenção do câncer de colo uterino ${ }^{(14,15)}$.

Ao verificar o conhecimento das mulheres sobre o exame de Papanicolau, o resultado foi satisfatório, pois a maioria demonstrou conhecer a finalidade do exame. Todavia, reconhecer a importância do exame não é o fator primordial ou decisivo para as mulheres se submeterem a esse procedimento. É preciso uma disposição e uma convicção pessoal, uma vontade interior capaz de se sobrepor à insegurança e outros bloqueios, possibilitando o ato voluntário de ir ao encontro da prevenção ${ }^{(13)}$.

Quanto às dificuldades encontradas, observou-se que a mulher encontra várias barreiras prejudicais para a realização de uma prevenção correta e eficaz, pois o fato de expor seu corpo a faz sentir-se constrangida. Para as mulheres, o sentimento de vergonha está diretamente relacionado à impessoalidade do procedimento envolvendo a exposição do corpo e a sua sexualidade ${ }^{(1)}$.

A relação que a mulher tem com sua sexualidade interfere diretamente na maneira como ela reage ao exame que manuseia órgão e região genital. $\mathrm{O}$ sexo tem fator decisivo na relação do indivíduo consigo e com suas relações interpessoais. Quando uma mulher não é bem orientada, essa exposição dos órgãos genitais pode gerar vergonha e conflitos sociais ${ }^{(16)}$.

A exposição ginecológica gera desconforto e constrangimento. O profissional de saúde é visto como um fiscal e juiz. A sensação de impotência e desproteção ao assumir a posição ginecológica e se deixar invadir pelo instrumental ou pelo toque ginecológico produz na mulher sensações de vergonha, medo e constrangimento, embora ela reconheça como necessária a realização do exame ${ }^{(16)}$.

Existem outros fatores que não podem ser negligenciados pelos profissionais de saúde, descritos em outras pesquisas da mesma natureza, como a falta de tempo, o medo, a ausência de sintomas, ou o fato de a cliente não gostar do profissional que realiza o procedimento. Nesse sentido, a educação em saúde é a melhor forma de aproximar as mulheres desse procedimento e, consequentemente, contribuir para a prevenção do câncer de colo uterino ${ }^{(17)}$.

Assim, o presente estudo detectou um número considerável de mulheres com comportamento que as tornam vulneráveis à doença: multíparas, com iniciação sexual precoce, além de baixo nível socioeconômico e baixo grau de escolaridade. Além dos fatores socioeconômicos, existem ainda os fatores impeditivos da realização do exame preventivo, como o sentimento de vergonha, medo e ansiedade.

Dessa forma, compreende-se que é preciso repensar a prática de saúde no sentido de expor procedimentos e orientações não somente técnicos, mas também adotar uma postura compreensiva, entendendo sua cultura e vivências. Nesse contexto, merecem destaque as medidas educativas e de informação em saúde. As informações à população contribuirão para uma nova postura diante das mudanças comportamentais, levando à prevenção de doenças de uma forma mais consciente e eficaz, possibilitando às mulheres uma melhor qualidade de vida.

\section{CONCLUSÃO}

Apesar de a maioria das mulheres realizar o exame periodicamente, muitas desconhecem a sua verdadeira finalidade, sentindo-se envergonhadas e constrangidas durante a realização do exame.

\section{REFERÊNCIAS}

1. Sepúlveda VP, González CF, Napolitano RC, Roncone DE, Cavada CG. Câncer de Cuello Uterino: Sobrevida a 3 y 5 años em Hospital San José. Rev Chil Obstet Ginecol. 2008;73(3):151-4.

2. Instituto Nacional de Câncer José Alencar Gomes da Silva - INCA. Estimativa 2012: incidência de câncer no Brasil. Rio de Janeiro: INCA; 2012.

3. Instituto Nacional de Câncer José Alencar Gomes da Silva - INCA. Diretrizes brasileiras para o rastreamento do câncer do colo do útero. Rio de Janeiro: INCA; 2011.

4. Ministério da Saúde (BR), Secretaria de Atenção à Saúde, Departamento de Atenção Básica. Controle dos cânceres do colo do útero e da mama. Brasília: Ministério da Saúde; 2013.

5. Carvalho MCMP, Queiroz ABA. Lesões precursoras do câncer cervicouterino: evolução histórica e subsídios para consulta de Enfermagem ginecológica. Esc Anna Nery Rev Enferm. 2010;14(3):617-24. 
6. Garcia CL, Pereira HC, Marinho MNASB. Percepções das mulheres acerca do exame de prevenção do câncer cérvico-uterino. Rev Bras Promoç Saúde. 2010;23(2):118-25.

7. Instituto Nacional de Câncer José Alencar Gomes da Silva - INCA. Programa Nacional de controle do câncer do colo do útero [acesso em 2014 Abr 9]. Disponível em http://www2.inca.gov.br/wps/wcm/connect/acoes programas/site/home/nobrasil/programa_nacional_ controle_cancer_colo_utero/deteccao_precoce

8. Albuquerque ZBP, Tavares SBN, Manrique EJC, Souza ACS, Neves HCC, Valadares JG, et al. Atendimento pelo SUS na percepção de mulheres com lesões de câncer cervicouterino em Goiânia-GO. Rev Eletrônica Enferm [periódico na Internet]. 2011 [acesso em 2014 Jun 25]; 13(2):239-49. Disponível em: http://www.fen. ufg.br/revista/v13/n2/v13n2a10.htm

9. Mascarello KC, Zandonade E, Amorim MHC. Análise da sobrevida de mulheres com câncer do colo do útero atendidas em hospital de referência para oncologia no Espírito Santo, Brasil, nos anos de 2000 a 2005. Cad Saúde Pública. 2013;29(4):823-31.

10. Eduardo KGT, Moura ERF, Nogueira PSF, Costa CBJS, Pinheiro AKB, Silva RM. Conhecimento e mudanças de comportamento de mulheres junto a fatores de risco para câncer de colo uterino. Rev Rene. 2012;13(5):1045-55.

11. Silva GA, Gamarra CJ, Girianelli VR, Valente JG. Tendência da mortalidade por câncer nas capitais e interior do Brasil entre 1980 e 2006. Rev Saúde Pública. 2011;45(6):1009-18.

12. Chatenoud L, Bertuccio P, Bosetti C, Levi F, Curado MP, Malvezzi M, et al. Trends in cancer mortality in Brazil, 1980-2004. Eur J Cancer Prev. 2010;19(2):7986.
13. Ferreira MLSM. Motivos que influenciam a nãorealização do exame de Papanicolaou segundo a percepção de mulheres Conhecimento das mulheres sobre o câncer cérvico-uterino. Esc Anna Nery Rev Enferm. 2009;13(2):378-84.

14. Matão MEL, Miranda DB, Campos PHF, Machado AF, Ornelas ER. Percepção de mulheres acerca do exame colpocitológico. Rev Enferm Cent.-Oeste Min. 2011;1(1):47-58.

15. Azevedo SG, Girianelli VR, Gamarra CJ, BustamanteTeixeira MT. Cervical cancer mortality trends in Brazil, 1981-2006. Cad Saúde Pública. 2010;26(12):2399407.

16. Fonseca LAM, Eluf-Neto J, Wunsch Filho V. Cancer mortality trends in Brazilian state capitals, 19802004. AMB Rev Assoc Med Bras. 2010;56(3):309-12.

17. Oliveira WMA, Barbosa MA, Mendonça BOM, Silva AA, Santos LCF, Nascimento LCD. Adesão de mulheres de 18 a 50 anos ao exame colpocitológico na estratégia saúde da família. Referência. 2012;3(7):1522.

\section{Endereço do primeiro autor:}

Alanda Maria Rodrigues Santos

Universidade Federal de Alagoas (UFAL)

Avenida Lourival Melo Mota, S/N

Cidade Universitária

Bairro: Tabuleiro dos Martins

CEP: 57072-900 - Maceió - AL - Brasil

E-mail: alanda.rodrigue@hotmail.com

\section{Endereço para correspondência:}

Amuzza Aylla Pereira dos Santos

Universidade Federal de Alagoas (UFAL)

Avenida Lourival Melo Mota, S/N - Cidade Universitária

Bairro: Tabuleiro dos Martins

CEP: 57072-900 - Maceió - AL - Brasil

E-mail: amuzasantos@bol.com.br 